\title{
Eye casualty services in London
}

\begin{abstract}
The combined pressures of the European Working Time Directive, $4 \mathrm{~h}$ waiting time target, and growing rates of unplanned hospital attendances have forced a major consolidation of eye casualty departments across the country, with the remaining units seeing a rapid increase in demand. We examine the effect of these changes on the provision of emergency eye care in Central London, and see what wider lessons can be learned. We surveyed the managers responsible for each of London's 8 out-of-hours eye casualty services, analysed data on attendance numbers, and conducted detailed interviews with lead clinicians. At London's two largest units, Moorfields Eye Hospital and the Western Eye Hospital, annual attendance numbers have been rising at $7.9 \%$ per year (to 76034 patients in $2010 / 11$ ) and $9.6 \%$ per year (to 31128 patients in 2010/11), respectively. Using Moorfields as a case study, we discuss methods to increase capacity and efficiency in response to this demand, and also examine some of the unintended consequences of service consolidation including patients travelling long distances to geographically inappropriate units, and confusion over responsibility for out-of-hours inpatient cover. We describe a novel 'referral pathway' developed to minimise unnecessary travelling and delay for patients, and propose a forum for the strategic planning of London's eye casualty services in the future.
\end{abstract}

Eye (2013) 27, 320-328; doi:10.1038/eye.2012.297; published online 1 February 2013

Keywords: eye casualty; paediatric eye casualty; ophthalmic accident and emergency

HB Smith, Eye Casualty Department, Moorfields Eye Hospital, 162 City Road, London EC1V 2PD, UK. Tel: + 44 (0)20 72533411 ; Fax: + 44 (0)20 72534696 E-mail: hbs@doctors.net.uk

Received: 29 February 2012 Accepted in revised form: 16 December 2012 Published online: 1 February 2013
HB Smith, CS Daniel and S Verma

Europe. ${ }^{2}$ It outlines the effects of organisational change and ever increasing demand on these services, and uses London's largest eye casualty department at Moorfields Eye Hospital (Moorfields) as a case study to explore how providers can respond to these pressures.

The delivery of emergency eye care in London has changed considerably over the last decade, reflecting both government policy and shifting clinical demands. The 'NHS Plan' published in July 2000 marked a turning point in the provision of accident and emergency (A\&E) care, with the introduction of the $4 \mathrm{~h}$ target and a significant increase in resources being directed towards the service. ${ }^{3}$ At Moorfields, as with eye casualty departments elsewhere, this has help drive the transformation of emergency eye care from a 'Cinderella service' into a properly resourced subspecialty.

In addition to the service provided by GPs and optometrists, emergency eye care in London is delivered in three main hospital settings: dedicated eye casualty departments, rapid access outpatient clinics, and general A\&E departments. Rapid access outpatient clinics tend to operate limited opening hours (normally standard weekday clinical sessions) and with limited capacity. Some provide a walk-in service, though others operate on a referral-only basis. Dedicated eye casualty departments typically offer a walk-in service, but London now has only two centres providing full 24 h-a-day, 7 day-a-week cover (Moorfields and the Western Eye Hospital). A further six units provide an out-of-hours on-call ophthalmic service for eye casualties attending through general A\&E (Royal Free Hospital, The Royal London Hospital, St Thomas' Hospital, King's College Hospital, Whipps Cross University Hospital and St George's Hospital). In these units, patients will typically see a casualty officer first, before onward referral to an ophthalmologist if required.

Existing literature concerning eye casualty services has typically focussed on surveys of the 
clinical presentations, demographics, and appropriateness of casualty attendances, the treatment of specific ophthalmic emergencies, and on the role of nurse practitioners in the delivery of emergency eye care. Relatively little has been published on the subject of the structure and logistics of service provision, and as such this article is largely dependent on the direct experience of its authors and contributors rather than on reference to existing literature.

The need for an emergency ophthalmic service is generally accepted, though some studies have concluded that the relative rarity of genuinely urgent attendances out-of-hours might justify a shift from a 24-h walk-in service to an on-call service accessed through general A\&E outside normal working hours. ${ }^{4}$ Others have suggested that ophthalmic primary care centres staffed by GPs, optometrists and nurse practitioners might provide a suitable alternative to the ophthalmologist-led services more typical in Central London. ${ }^{5}$ These recommendations reflect an acknowledgement of the more widespread view that eye casualty often acts as a primary care service rather than a true emergency service. ${ }^{6}$

There is also an impression from the literature, confirming the experience of the authors and contributors to this paper, that eye casualty services routinely run in excess of their intended capacity. $4,6,7$

\section{Shaping today's Eye casualty provision}

Demand for eye casualty services has increased rapidly in recent years. Figure 1 illustrates attendance numbers for Moorfields and the Western Eye Hospital, London's two largest units. Volume has risen particularly fast over the last 5 years, averaging $7.9 \%$ per year at Moorfields, and $9.6 \%$ per year at the Western Eye Hospital. This has seen annual attendances increase from 51248 in 2005/06 to 76034 in 2010/11 at Moorfields, and from 21556 in 2005/06 to 31128 in 2010/11 at the Western Eye Hospital.

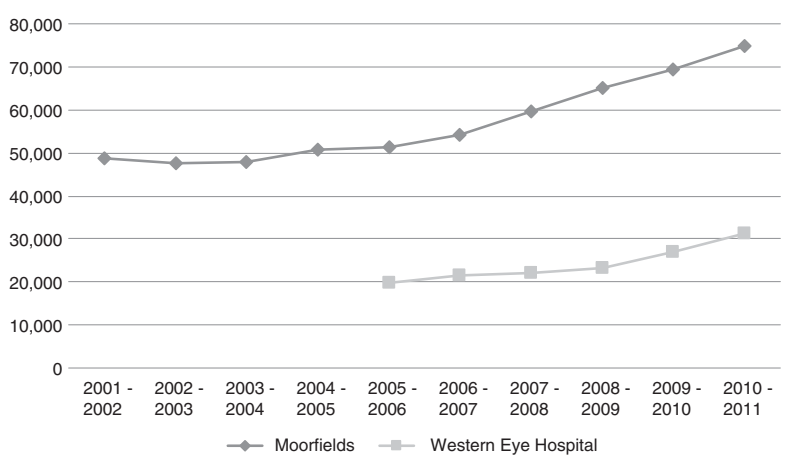

Figure 1 Eye Casualty Attendances at Moorfields and the Western Eye Hospital, 2001-2011.
These numbers are significant even in the context of large general A\&E departments. King's College Hospital, for example, London's busiest A\&E, saw 149902 patients in $2010 / 11 .^{8}$ If this rate of growth is maintained, then Moorfields will be seeing 100000 casualty patients per year by 2015, and the Western Eye Hospital 45000 per year, a doubling of demand over 10 years.

Three main factors appear to account for this trend: the need to consolidate out-of-hours care into fewer, larger units, which have consequently become busier; a generalised increase in unplanned hospital attendances; and London's growing population.

\section{Consolidation}

In common with other specialties such as otolaryngology and dermatology, ophthalmology has seen a considerable consolidation of services over the last 10 years. This has largely been driven by the need to comply with the European Workings Times Directive (EWTD) and the European Court of Justice ruling on on-call working (October 2000). The former requires $11 \mathrm{~h}$ of continuous rest in each $24 \mathrm{~h}$ period, and a maximum working week of $48 \mathrm{~h}$. The latter defines hours spent 'on-call' as 'work'. The net effect of these regulations, which were phased in between 2004 and 2009, was to render smaller units unsustainable. ${ }^{9}$ As a result, resources have been pooled so that out-of-hours cover is provided by fewer doctors who are working in larger units, but which are consequently busier.

\section{Increased unplanned hospital attendance}

In 2009/10 20.5 million people attended A\&E departments in England, up by 5\% on the previous year, and an increase from 14 million in 2002/3 (after two decades of relative stability). ${ }^{3}$ A number of factors are thought to have contributed to this change including confusion over GP out-of-hours services, 3,10 difficulty in obtaining GP appointments, a growing immigrant population who tend not to register with family doctors, ${ }^{11}$ and the reduction in A\&E waiting times making it a more convenient alternative. ${ }^{3}$ In the case of specialist services such as eye casualty departments, there may also be the view among patients that a specific disorder would be best diagnosed and managed by a specialist in that field, and they, therefore, elect to access these services directly, bypassing their GP. ${ }^{12}$

In May 2005, Moorfields undertook a prospective questionnaire-based survey of 560 consecutive patients attending eye casualty to investigate the appropriateness of attendances. ${ }^{13} 51.9 \%$ of attendees were men, and the median age was 35 years. Four-hundred and thirty-one cases $(76.9 \%)$ were self-referrals, $56(10 \%)$ were referred 
by community optometrists, $45(8.1 \%)$ were referred by GPs, and 28 (5\%) were referred by other hospitals. Onehundred and seventy-one cases $(30.6 \%)$ were considered to be non-acute, and 210 cases $(37.5 \%)$ were considered suitable for a GP or optometrist to see. Three-hundred and fifty-two patients $(62.8 \%)$ had symptoms for less than a week, $144(25.7 \%)$ for between a week and a month, and $64(11.5 \%)$ for more than a month before presentation. Three-hundred and fifty patients $(62.5 \%)$ were discharged on the day of presentation. The reasons stated by the patients for their attendance included: 'great concern' (66.2\%), 'convenience' (11.8\%), 'second opinion' (8.2\%), 'couldn't wait for GP appointment' (3.2\%), and 'other' $(10.6 \%)$. The authors concluded that despite a desire to encourage more appropriate use of primary care services for 'non-urgent' complaints, the high anxiety levels associated with the fear of loss of vision, and the disfiguring nature of some eye conditions such as conjunctivitis and chalazion, represented a major hurdle in influencing attendance behaviour. Although unpublished, this survey has recently been repeated as part of an internal service appraisal with broadly similar results. These findings are in keeping with previous studies, which report rates of self-referral of between 54-89.9\%, with the median around $80 \%$. Other surveys have typically found an even greater male preponderance among attendees (perhaps reflecting an under representation of manual labour, farming, and manufacturing in Central London), but the case mix is otherwise broadly representative. $4,6,7,14,15$

\section{Changing demographics}

London's population has grown every year since 1988 and the Office of National Statistics' most recent estimate for the population of Greater London is 7825177 (June 2010), up from 7065500 in 1998, a rise of $10.1 \%$ over 12 years. ${ }^{1}$ In addition to London's 7.8 million residents there is also a large transitory population with an estimated one million commuters travelling to the capital each working day, ${ }^{16}$ and a further 26 million tourists visiting each year. ${ }^{17}$ Commuters and visitors are much more likely to use emergency services than permanent residents. ${ }^{18}$

Users of emergency medical services also tend to be young relative to the general population, ${ }^{19}$ and London has a young population compared with other regions in the UK. ${ }^{20}$ Thirty-one percent of Londoners are under 24-years-old and London's birth rate is one of the highest in the country. ${ }^{21}$ London also continues to attract a large, young, mobile working population, which is likely to perpetuate this demographic trend.

\section{The Moorfields experience}

Although operating one of the largest ophthalmic emergency services in the world, most of the pressures faced by Moorfields are common to any large eye casualty department. As such, many of the changes to the service that have been implemented to respond to these pressures are representative of similar changes made by units across London and the country as a whole. Using Moorfields as an example, we examine some of the steps that have been taken to increase capacity and improve efficiency in an effort to meet patient expectation and demand.

However, it is first worth considering some of the factors that may make Moorfields rather atypical. The first is name recognition, encouraged by a deliberate 'branding' policy adopted by the hospital in recent years. ${ }^{22}$ As well as the main facility at City Road, there are now 18 further Moorfields-run and Moorfieldsbranded satellite units across the capital, a number which is likely to rise further in the near future. While most of the early satellite units were situated within hospitals, the number of community-based locations has recently increased. Moorfields now runs an ophthalmic service in London's first purpose-built polyclinic in Loxford, and is currently involved in a pilot project with high-street opticians chain Boots in Watford. In Harrow, Moorfields has have joined forces with a local group of GPs to provide more eye care in the community. The net effect is that over $50 \%$ of London's population is now served by a Moorfields-run ophthalmic service, ${ }^{22}$ and many of these patients understandably think of Moorfields first in the event of an ophthalmic emergency. This familiarity may also be reflected in the referral behaviour of GPs and community optometrists.

Another causal factor for the rapid increase may be the guaranteed access offered by Moorfields. In recent years some other large London units have been forced to temporarily close their doors to new casualty attendances due to lack of capacity. While this has previously been commonplace for some subspecialty services such as vitreoretinal surgery, it has historically been a rarity for general ophthalmic emergencies. To date Moorfields, in common with the Western Eye Hospital and other large metropolitan eye units in the United Kingdom, has managed to maintain a policy of never refusing a patient on the grounds of capacity. This may have encouraged referrals from other healthcare professionals who had previously experienced difficulty in accessing ophthalmic care for their patients in more local units.

\section{Maintaining the service}

It should be remembered that an eye casualty service can be a valuable source of revenue for a hospital. Not only do patients attract a tariff for their casualty attendance, but for the third of patients who require follow-up additional hospital episodes will also generate income. 
This could potentially lead to a conflict of interest. On the one hand there is a positive financial incentive to promote attendances; on the other hand there is the challenge of diverting manpower, diagnostic resources, and physical space away from elective services, together with the need for additional outpatient capacity to accommodate the follow-up visits generated.

Many of the factors responsible for recent increases in demand look set to continue, and the remaining eye casualty services in London face considerable challenges in responding to this rate of growth. In common with other units, Moorfields has addressed increased demand with increased capacity, but it has also sought to manage attendances more effectively by seeking to ensure that the patient's progress through the department is as efficient as possible.

\section{Increased capacity}

The Moorfields eye casualty department has undergone considerable expansion both in terms of personnel and physical space.

Ten years ago Moorfields provided its first consultant cover in casualty for 3 sessions per week, with a total complement of four or five doctors providing the weekday 0900-1700 hours service. Out-of-hours cover was provided by two doctors (a resident junior registrar known as 'Lower House', and an on-call senior registrar known as 'Upper House'). In addition, one on-call consultant was available. There are now 1 or 2 consultants present for every weekday daytime shift (18 sessions in total), supported by an average of seven additional junior doctors. In the evenings the Upper House registrar is supported by three additional doctors until 2100 hours. Overnight cover is still provided by a single Lower House registrar, though activity has increased considerably, and with on-call support from the Upper House who is also responsible for providing inpatient cover to a number of satellite units (Northwick Park, Ealing, and Great Ormond Street). On-call cover has also been expanded to include a fellow and consultant for each subspecialty. At the weekends staggered daytime shifts provide five doctors at peak times on Saturdays, and four on Sundays.

The department has also sought to make increased use of other care providers such as nurse practitioners, optometrists, and GP. They have now become a critical part of the service, and will be discussed in detail in the following section.

The Western Eye Hospital has responded similarly, and has recently introduced a regular consultant presence in eye casualty (currently four sessions per week), increased doctor numbers to three per weekday daytime shift, and trained three specialist ophthalmic nurse practitioners (providing at least one nurse practitioner for most daytime shifts). In addition, the Western Eye Hospital aims to provide same-day minor surgery to casualty attendees.

\section{Increased efficiency}

Every effort has been made to reduce delays to the patients' progress through the department, and to maximise the efficient use of the doctors' time for diagnosis and management. In common with other eye units, this includes the use of nursing staff to take the initial ophthalmic and general medical history, to undertake testing of visual acuity, and other initial investigations such as pupil reactions and colour vision, and to instil dilating drops when required. Ancillary investigations such as optical coherence tomography, ultrasonography, CT, and visual fields are also readily available, as are allied professionals such as orthoptists.

Recently efforts have been made to reduce the number of patients booked for follow-up in the casualty department. Strict guidelines, and a requirement to agree all casualty reviews with a consultant, have been successful in easing the pressure placed on the casualty service by inappropriate follow-up. In part, this has been facilitated by the provision of rapid access primary care clinics, as well as a number of new subspecialty urgent care clinics in medical retina, external diseases, and neuro-ophthalmology. These complement an already well-established vitreoretinal emergency service, which runs in parallel with the general eye casualty, and which also acts as a regional tertiary-referral unit. The Western Eye Hospital has similarly expanded its provision of subspecialty rapid access follow-up clinics.

Major investment has been made in the provision of improved information technology support at Moorfields, with the introduction of the OpenEyes system. This will streamline clinical record keeping, generate GP letters and prescriptions, and will also enable the discharging doctor to book follow-up appointments directly. The equivalent system at the Western Eye Hospital, Symphony, has been in place for several years. The networking of imaging has also been improved, and it is now possible to view OCTs, fluorescein angiograms, and photographs in eye casualty. In addition, it is possible to access radiology images and reports not only for scans performed at Moorfields, and also for imaging sent from outside hospitals.

\section{Escalation procedures}

Both Moorfields and the Western Eye Hospital have developed a series of escalation procedures to deal with unexpected surges in activity. A variety of techniques are 
employed, including offering non-urgent patients the option of returning to a primary care clinic at a later date, asking clinical and clerical staff to extend shifts or start shifts early, bringing in locum staff, and diverting casualty attendees to outpatient clinics.

\section{Telephone triage}

For many years, Moorfields has provided a telephone advice service for patients and healthcare professionals. In common with other aspects of the service it has seen demand increase considerably. The call operators can advise patients as to whether they need to be seen, and if so where, can offer advice on the treatment of simple eye complaints, and can advise other healthcare professionals on the appropriate referral of their patients.

\section{Referral pathways}

In response to the trend for patients to come to Moorfields from ever greater distances, the casualty department has recently developed a referral pathway to try to ensure that patients are directed to the most geographically-appropriate unit. This is intended to minimise unnecessary travel and delay in patients obtaining their initial consultation, and should also help to ensure any subsequent follow-up is in the most suitable local department.

The referral pathways follow the established 'hub-andspoke' model of eye care provision in London. This exists on three tiers. The first tier consists of the small community-based eye departments, which provide either no casualty service at all, or a small number of slots in existing clinics booked on an ad hoc, referral-only basis. The second tier consists of the larger units, typically based in district general or teaching hospitals, which provide dedicated eye casualty services usually on a walk-in basis, but typically only during 'office hours'. The third consists of the larger regional units discussed in the introduction, which provide a 24-h service either in a dedicated eye casualty department or via general A\&E with an on-call ophthalmologist. These 'hub' services are normally staffed by a rota of doctors supplied by the 'spoke' units they cover.

We have found that the nature of the relationship between the 'hub' and 'spoke' units in providing out-ofhours outpatient cover can vary considerably from location to location. In the case of Moorfields, the 'spoke' units it serves are typically Moorfields satellites sharing common centralised management and personnel. For other 'hubs', the 'spoke' units have formed cooperatives, for example the South West London Ophthalmology Network with its eye casualty based at St George's Hospital, Tooting. Elsewhere more informal arrangements exist between units, and in some cases there is no officially-agreed cover at all.

To encourage the appropriate use of the 'hub-andspoke' model, Moorfields has developed a map (Figure 2) and referral pathway table (Figure 3). Although originally intended for internal use only-to address the specific problem of delay, cost, and inappropriate follow-up experienced by patients travelling long distances to Moorfields-other units may also find this approach useful. Although every effort has been made to ensure the accuracy of the information in these pathways, we occasionally encountered uncertainty even within the units themselves about the exact commitments of their eye casualty cover.

\section{Nurse practitioners, GPs, and optometrists in eye casualty}

Nurse-led care pathways are already widespread in ophthalmology, particularly in the preoperative and postoperative assessment of cataract patients. However, nurse-led care also has a major role to play in eye casualty, and their contribution is central to the service provided at Moorfields, where they are known as ophthalmic emergency nurse practitioners or ENPs. Becoming an ENP is not a formal qualification, although additional training is expected. A number of external courses are available to provide this, though increasingly Moorfields undertakes this training internally. The ability of nurses to provide a safe and effective service in the management of eye casualties has been demonstrated by several studies elsewhere, ${ }^{23-26}$ and confirmed by internal audit at Moorfields.

Following triage and initial visual assessment (which is undertaken by nurses for all patients attending eye casualty), patients with suitable conditions are directed to the ENP pathway where they are examined, treated, and discharged by a nurse according to a protocol, and without further reference to a doctor unless the diagnosis is in doubt. The disorders presently covered are blepharitis, chalazion, corneal abrasion, bacterial conjunctivitis, viral conjunctivitis, subconjunctival haemorrhage, trichiasis, retained contact lens, subtarsal foreign body, arc eye, corneal foreign body, and recurrent erosion syndrome. These diagnoses currently constitute $32 \%$ of presentations to Moorfields eye casualty (internal departmental audit, July 2012), and the existing ENP service comprises a pool of 25 appropriately trained nurses, 3 of whom staff each session providing cover 08:00-16:30 on weekdays, and seeing $17 \%$ of casualty attendances overall. Over the next few years Moorfields plans to recruit an additional three ENPs supported by a further two healthcare assistants and allowing the service to be extended to 08:00-21:00, seven days per week. 


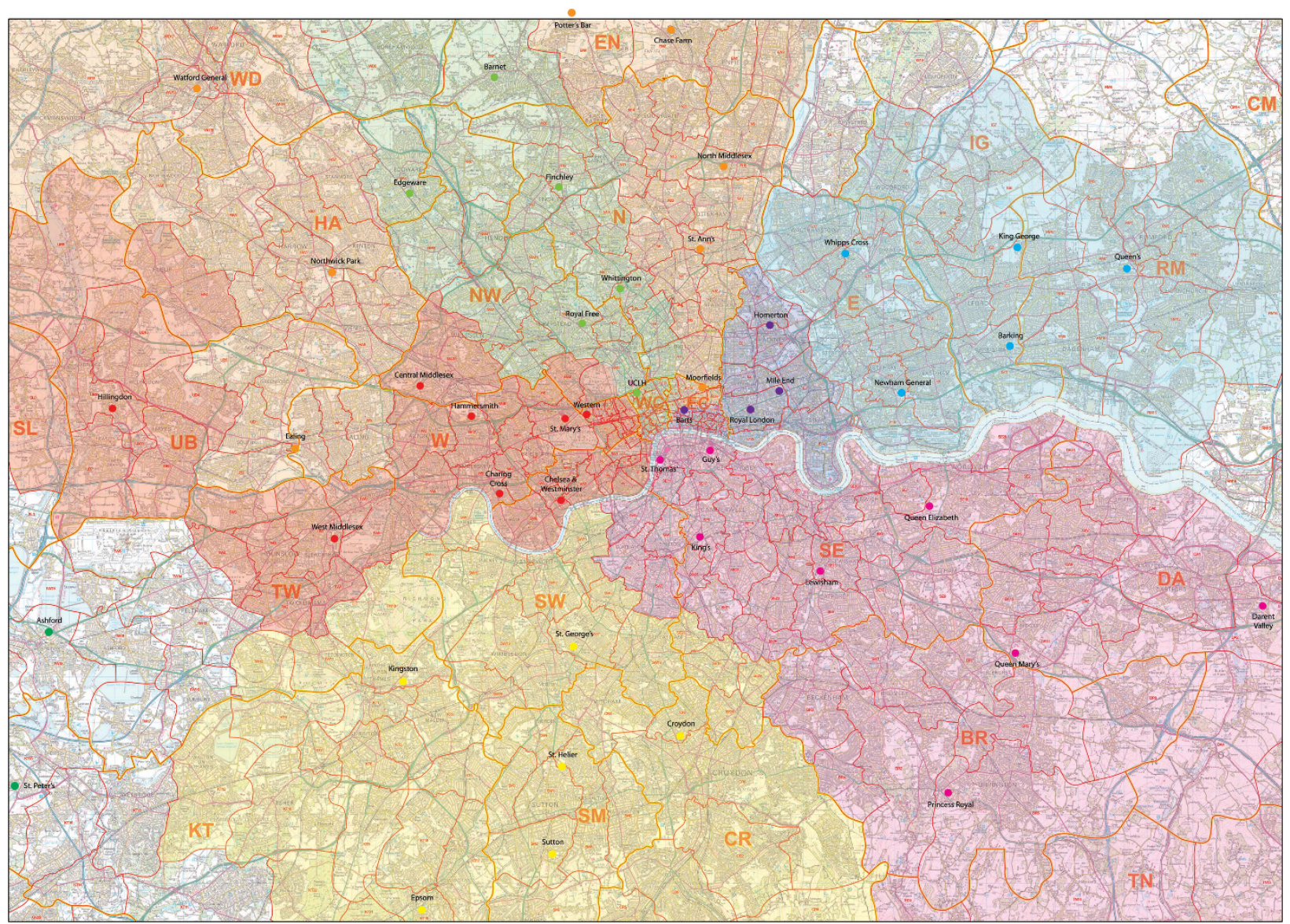

Figure 2 Referral Postcode Map.
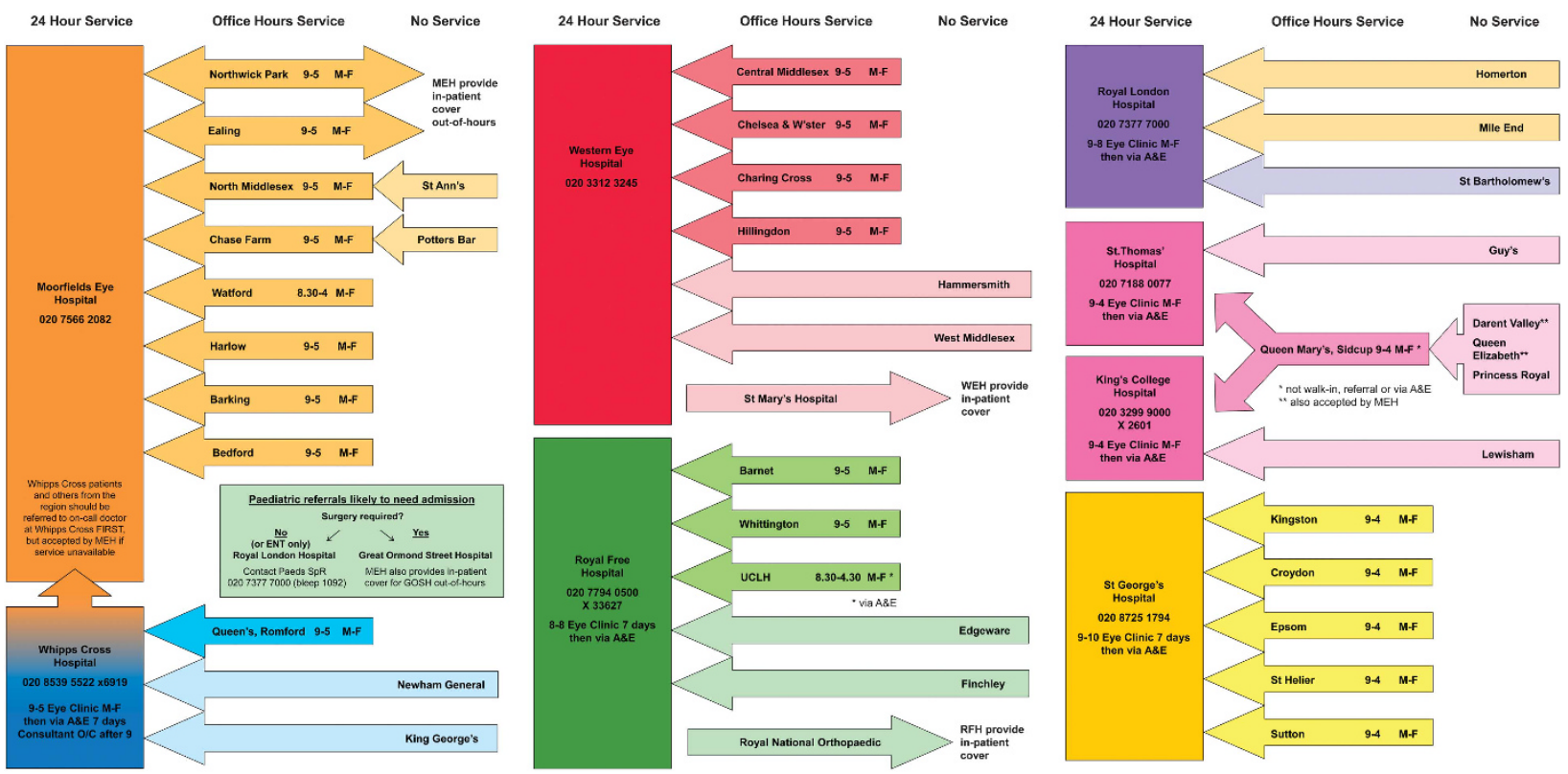

Figure 3 Referral Pathway Table. 
Since 2006 nurses have had the opportunity of becoming 'independent prescribers' following additional training. Nurse prescribers are able to prescribe any drug (including unlicensed drugs and controlled drugs) under the same terms as a medical practitioner, and 'within their own level of experience and competence' ${ }^{27}$ They remain relatively uncommon (constituting $2-3 \%$ of the nursing workforce), and Moorfields do not currently employ any independent nurse prescribers in eye casualty. However, an additional scheme known as a 'Patient Group Directive' (PGD) allows for the delegation of limited prescribing powers to nurses who are not independent prescribers, for the treatment of specified groups of patients with specific diagnoses according to a protocol. A PGD consists of a local agreement between nurses, management, pharmacy and the lead clinician, which must be renewed annually, and which requires the verbal consent of patients. The PGDs in place in Moorfields eye casualty cover the diagnoses listed above, and the prescribing of, when appropriate,

chloramphenicol $1 \%$ ointment, chloramphenicol $0.5 \%$ drops, and hypromellose $1 \%$ drops.

Several factors have driven the development of the ENP programme at Moorfields. First, the ENP pathway frees up doctors to see more serious problems. Second, it allows patients with minor complaints to be seen more speedily, reducing waiting times, and improving the overall patient experience. Third, the ENP pathway has proven itself to be a safe and effective means of providing additional capacity within the casualty department. Fourth, the ENP service has made staffing casualty easier by reducing the reliance on locum doctors, the availability of whom cannot always be relied upon. Fifth, the additional expertise and responsibility that comes with ENP status has been popular with nursing staff, providing an opportunity for career progression for senior nurses within the department. Although ENP status does not in itself carry a salary uplift, it does provide opportunities for further bank work. Sixth, the move to nurse-led care has been encouraged by service commissioners as part of a wider strategy to separate primary and emergency care services, and to ensure cost-effectiveness.

In addition to ophthalmologists and ENPs, general practitioners and optometrists are also used to deliver emergency eye care, both within their own practices and as members of an eye casualty department. One GP with a special interest in ophthalmology is employed by Moorfields, along with two optometrists, each currently work one session per week in eye casualty. It is understood that other units, particularly in Wales, use optometrists in the delivery of eye casualty services far more extensively. In addition to the core training that optometrists receive in the diagnosis of ocular disease, they have similar opportunities to nurses for obtaining independent prescriber status.

\section{Paediatric eye casualty}

Providing a paediatric eye casualty service presents some particular challenges, especially for dedicated eye hospitals such as Moorfields and the Western Eye Hospital. For 'office hours' cover, the Care Quality Commission requires a multidisciplinary team, which must include paediatric-trained specialist nurses, play leaders, counsellors, and facilities including segregated paediatric waiting and treatment areas, and subspecialty paediatric clinics. These requirements have proven very difficult for some units to deliver. Moorfields is able to provide an 'office hours' service via a dedicated paediatric eye hospital in an adjoining building, the Richard Desmond Children's Eye Centre. This is the largest eye hospital in the world devoted solely to the treatment of children.

However, for 'out-of-hours' services Moorfields and the Western Eye Hospital are both heavily reliant on robust network relationships with other units able to provide general paediatric input, and/or paediatric admissions. Both units have a strict policy of not accepting children who are systemically unwell or likely to require admission. In the case of Moorfields, children needing mainly medical treatment or non-ophthalmic surgery are directed to the Royal London Hospital, while those likely to need ophthalmic surgery are sent to Great Ormond Street Hospital. Once there they would be seen by the Upper House on-call or by the on-call consultant paediatric ophthalmologist. In the case of the Western Eye Hospital, unwell children or those likely to require admission are sent to St Mary's Hospital. Other eye casualty departments operating from large general hospitals will typically have easier access to paediatric medical cover.

Plans are currently underway to develop a specialist out-of-hours paediatric eye casualty at the Royal London Hospital, which will become a hub for the cover currently provided by paediatric ophthalmologists from Moorfields, the Royal London Hospital and Great Ormond Street Hospital.

\section{Conclusions}

In common with other large eye casualty departments across London, Moorfields has witnessed a considerable increase in demand, seeing attendances rise by $7.9 \%$ year-on-year. This reflects the combined pressures of reduced service provision by smaller units, increased demand from a growing population, and rising patient 
expectations in an increasingly $24 \mathrm{~h}$-a-day, 7 day-a-week society.

From the patient's perspective, this trend offers potential benefits as well as disadvantages. The concentration of expertise and diagnostic facilities at large, specialist units can provide an excellent standard of care, particularly for those suffering from rare or difficult conditions, or where prompt emergency treatment is important. The cost, however, is that the majority of patients with relatively minor complaints often travel much further than is necessary, and can be asked to attend inappropriately located follow-up appointments or experience poor continuity of care. At Moorfields the introduction of maps and referral pathway tables has been useful in allowing staff to ensure that patients referred by phone are directed to the most appropriate local unit. However, it should be stressed that the priority is convenience for the patient, and any patient expressly wishing to attend Moorfields will never be turned away.

Within the remaining eye casualty departments the ever present pressures to increase capacity and improve cost-effectiveness are driving innovation. The growing use of allied professionals such as nurse practitioners and optometrists can free-up doctors to concentrate on the diagnosis and management of more challenging cases. This can deliver a service which is both time-efficient, high quality, and professionally fulfilling for those involved. Departments have also been forced to pay close attention to patient pathways in order to optimise efficiency, and to remove bottlenecks. Increased provision of subspecialty follow-up clinics, better access to diagnostic tests, and upgraded information technology are all examples of improvements, which have demonstrable benefits to productivity.

Over the last 10 years a number of pressures such as the EWTD and the $4 \mathrm{~h}$ target have led to a major consolidation of services as smaller units have become unsustainable. This withdrawal of service from local departments may have had several unforeseen consequences. The first is uncertainty on the part of local GPs and optometrists as to the appropriate destination for ophthalmic referrals. There is anecdotal evidence that larger well-known units such as Moorfields and the Western Eye Hospital have increasingly become the destination for geographically-inappropriate referrals. The second is a lack of provision for patients experiencing postoperative complications following surgery at smaller units who do not provide an out-ofhours emergency service. The management of conditions such endophthalmitis, high intraocular pressure, and wound dehiscence on behalf of other units is placing an increasing burden on the remaining out-of-hours services. The third, and potentially most concerning, is a failure of some hospitals to make proper provision for inpatient care following the withdrawal of local eye casualty cover. While some units have signed formal Service Level Agreements for out-of-hours cover, others have made more ad hoc arrangements. A survey conducted by Moorfields in the course of developing the referral pathways exposed a worrying informality in the relationship between some 'hub' and 'spoke' units. Often there was uncertainty about service obligations between departments, and on occasion even out-and-out disagreement over responsibility for ophthalmic emergency cover. Inevitably this has led to disputes, and has the potential to delay urgent treatment for patients.

A further concern is the suitability of the larger eye casualty departments that remain. There are currently eight big regional units in Greater London (the 'hubs' in the 'hub-and-spoke' model). However, the development of these services has often occurred in relative isolation, and there has been no London-wide strategic planning of ophthalmic emergency cover. As a consequence these units are not always in locations that are appropriate to the populations that they serve. Furthermore, many of these units occupy old hospital buildings with antiquated, inflexible facilities, and limited space. This has led several departments, including Moorfields, to actively consider moving to alternative sites.

Not only has the consolidation of services and rapid increase in demand seen over the last 10 years significantly increased the burden on the remaining eye casualty departments, but it has also reduced local availability for patients. We are concerned that uncertainties over responsibility for inpatient cover, and the informal nature of the relationship between some units in the 'hub-and-spoke' model may pose a risk if treatment is delayed as a result. We believe that there is a strong case for a more coordinated approach to providing emergency ophthalmic care in London. One option might be to establish a forum for representatives of the capital's eye casualty departments to meet, formalise current arrangements, and discuss a strategy for service development in the future.

\section{Conflict of interest}

The authors declare no conflict of interest.

\section{Acknowledgements}

We are grateful to Mr Declan Flanagan, Medical Director, Moorfields Eye Hospital; Miss Louise Molloy, A\&E Service Manager, Moorfields Eye Hospital; Mr Humayun Mian, A\&E Service Manager, Moorfields Eye Hospital; Mr Keith Khunpha, Senior Performance/Information Analyst, Moorfields Eye Hospital; Mr Chris Whiting, 
Information Manager, Moorfields Eye Hospital; Mr Graham Duguid, Consultant Ophthalmologist, Western Eye Hospital; and Miss Zena Rodriguez, A\&E Service Manager, Western Eye Hospital, for their assistance in providing attendance data, and details of service configurations.

\section{References}

1 Office of National Statistics. Estimated Population of London, Mid-Year 2010, http://data.london.gov.uk/ datafiles/demographics/ons-mye-population-totals.xls (accessed November 2011).

2 Eurostat: Population of European Cities. http://epp. eurostat.ec.europa.eu/tgm/table.do?tab = table\&init $=1 \&$ language $=$ en $\&$ pcode $=\operatorname{tgs} 00079 \&$ plugin $=1$ (accessed November 2011).

3 The King's Fund. Our Future Health Secured? A review of NHS funding and performance, September 2007. http://www.kingsfund.org.uk/ publications/our-future-health-secured (accessed November 2011).

4 Kadyan A, Sandramouli S, Caruana P. Utilization of an ophthalmic casualty - a critical review. Eye 2007; 21: 441-442.

5 Rao GP, McGalliard JN, Harding SP. Hospital-based primary care centres in ophthalmology. Eye 1997; 11(1): 1-2.

6 Fenton S, Jackson E, Fenton M. An audit of the ophthalmic division of the accident and emergency department of the Royal Victoria Eye and Ear Hospital, Dublin. Ir Med J 2001; 94(9): 265-266.

7 Jones NP, Hayward JM, Khaw PT, Claoue CMP, Elkington AR. Function of an ophthalmic 'accident and emergency' department: results of a six month survey. Br Med J (Clin Res Ed) 1986; 292: 188-190.

8 Department of Health. A\&E Attendance Statistics. www.dh.gov.uk/en/Publicationsandstatistics/Statistics/ Performancedataandstatistics / AccidentandEmergency/ DH_087973 (accessed November 2011).

9 Royal College of Ophthalmologists. European Working Time Directive, College Suggestions. www.rcophth.ac.uk/ core/core_picker/download.asp?id=410 (accessed November 2011).

10 Thompson C, Hayhurst C, Boyle A. How have changes to out-of-hours primary care services since 2004 affected emergency department attendances at a UK District General Hospital? A longitudinal study. Emerg Med J 2010; 27: 22-25.

11 Boden P, Rees P. Using administrative data to improve the estimation of immigration to local areas in England. $J$ R Statist Soc A 2010; 173(4): 707-731.

12 Kulu-Glasgow I, Delnoij D, de Bakkar D. Self-referral in a gatekeeping system: patients' reasons for skipping the general-practitioner. Health Policy 1998; 45(3): 221-238.

13 Hau S, Ioannidis A, Masaoutis P, Verma S. Patterns of ophthalmological complaints presenting to a dedicated ophthalmic accident \& emergency department: inappropriate use and patients' perspective. Emerg Med J 2008; 25: 740-744.

14 Edwards RS. Ophthalmic emergencies in a district general hospital casualty department. Br J Ophthalmol 1987; 71: 938-942.

15 Bhopal RS, Parkin DW, Gillie RF, Han KH. Pattern of ophthalmological accidents and emergencies presenting to hospitals. J Epidemiol Community Health 1993; 45(5): 382-387.

16 Transport for London. Travel in London, Key Trends and Developments, Report Number 1 www.tfl.gov.uk/assets/ downloads/corporate/Travel-in-London-report-1.pdf (accessed November 2011)

17 Visit London. Key Visitor Statistics, 2010 Fact Sheet http:// vlstatic.com/l-and-p/assets/media/factsheet_key_ visitor_statistics_2010.pdf (accessed November 2011).

18 Jankowski RF, Mandalia S. Comparison of attendance and emergency admission patterns at accident and emergency departments in and out of London. BMJ 1993; 306: 1241-1243.

19 Downing A, Wilson R. Temporal and demographic variations in attendance at accident and emergency departments. Emerg Med J 2002; 19: 531-535.

20 Office for National Statistics. Summary: Regional Profiles, Population and Migration, London, October 2011. www.ons.gov.uk/ons/rel/regional-trends/region-andcountry-profiles/population-and-migration/populationand-migration-london.html (accessed November 2011).

21 Greater London Authority. Focus on London 2010, Population and Migration http://data.london.gov.uk/ datastorefiles/documents/FocusOnLondonPopulationAndMigration.pdf (accessed November 2011).

22 Moorfields Eye Hospital. A Vision of Excellence, A 10-year Strategy for Moorfields Eye Hospital www.moorfields.nhs.uk/portal_repository/files / OurVisionofExcellence-forwebsite.pdf (accessed November 2011).

23 Kirkwood BJ, Pesudovs K, Loh RS, Coster DJ. Implementation and evaluation of an ophthalmic nurse practitioner emergency eye clinic. Clin Experiment Ophthalmol 2005; 33(6): 593-597.

24 Banerjee S, Beatty S, Tyagi A, Kirkby GR. The role of ophthalmic triage and the nurse practitioner in an eyededicated casualty department. Eye 1998; 12(5): 880-882.

25 Bhatt R, Sandramouli S. Evidence-based practice in acute ophthalmology. Eye 2007; 21(7): 976-983.

26 Buchan JC, Saihan Z, Reynolds AG. Nurse triage, diagnosis and treatment of eye casualty patients: a study of quality and utility. Accid Emerg Nurs 2003; 11(4): 226-228.

27 Department of Health. Improving Patients' Access to Medicines: A Guide to Implementing Nurse and Pharmacist Independent Prescribing within the NHS in England, Department of Health, April 2006. http://www.dh.gov.uk/ prod_consum_dh/groups/dh_digitalassets/@dh/@en/ documents/digitalasset/dh_4133747.pdf (accessed October 2012). 